\title{
1 Has the income of the residential area impact on the use of intensive
}

2 care?

3

Janne H. Liisanantti ${ }^{1,2}$, Riikka Käkelä*1,2, Lasse V. Raatiniemi*2,3, Pasi Ohtonen ${ }^{2,4}$, Siiri Hietanen ${ }^{1,2}$, Tero I. AlaKokko $^{1,2}$

6

7 Oulu University Hospital, Department of Anesthesiology, Division of Intensive Care Medicine ${ }^{1}$, Oulu

8 University, Medical Research Center, Research group of Surgery, Anesthesiology and Intensive care², Oulu

9 University Hospital, Centre of Pre-Hospital Emergency Care ${ }^{3}$, and Oulu University Hospital, Division of

10 Operative Care ${ }^{4}$

Oulu University Hospital, P.O.BOX 21, 90029 OYS, Oulu, Finland

*Equal contribution

Intensive care and socioeconomic factors

2511 words

None of the authors have conflict of interrest

Corresponding author Janne H. Liisanantti

Oulu University Hospital

Department of Anesthesiology

Division of Intensive Care Medicine

P.O.BOX 21

90029 OYS

Oulu, Finland

janne.liisanantti@ppshp.fi 
Background: The socioeconomic factors have an impact on case mix and outcome in critical illness, but how these factors affect the use of intensive care is not studied. The aim of the present study was to evaluate the incidence of intensive care unit (ICU) admissions in patients from residential areas with different annual incomes.

Methods: Single-center, retrospective study in Northern Finland. All the non-trauma-related emergency admissions from the hospital district area were included. The postal codes were used to categorize the residential areas according to each area's annual median income: the low-

9 income area, $€ 18979$ to $€ 28841$ per year; the middle-income area, $€ 28879$ to $€ 33856$ per year; and the high-income area, €34 221 to $€ 53864$ per year.

Results: A total of 735 non-trauma-related admissions were included. The unemployment or retirement, psychiatric comorbidities and chronic alcohol abuse were common in this population. The highest incidence, 5.5 (4.6-6.7)/1000/year, was in population aged more than 65 years living in high-income areas. In working-aged population, the incidence was lowest in high income areas (1.5 (1.3-1.8/1000/year) compared to middle income areas (2.2 (1.9-2.6)/1000/year, $p=0.001)$ and low income areas (2.0 (1.7-2.4)/1000/, p=0.009) Poisonings were more common in low-income

17 areas. There were no differences in outcome. areas where the annual median income was below the median annual income of $€ 38775$ per inhabitant per year in Finland. 


\section{Introduction}

3 There are several studies that have investigated the relationship between socioeconomic factors

4 and critical illness or trauma. The main focus in previous studies has been the impact of

5 socioeconomic factors on outcome. They have shown that the causes of intensive care unit (ICU)

6 admissions vary depending on the social class or socioeconomic status. ${ }^{1-5}$ It has also been shown

7 that the outcomes of critically ill patients vary depending on socioeconomic factors and that the

8 patients from lower social classes seem to have higher mortality, but there are also controversial

9 results. ${ }^{1,2,6}$ To our best knowledge there are no previous studies on the impact of different

10 socioeconomic factors on the incidence of ICU admissions. Furthermore, most of the studies on

11 the impact that such factors have on outcome have been conducted in the United States and are

12 poorly generalizable to the Nordic countries with social insurance covered national health care.

13 Having more information about the role of income level in ICU mortality and the use of ICU resources is important when planning preventative interventions and organizing health resources.

15 The primary aim of the present study was to compare the incidence and causes of ICU admissions of non-trauma patients from residential areas with different income levels in one northern

17 university hospital district. In addition, we also compared outcome and the length of ICU stay 18 according to residential areas. 


\section{Setting}

3 The retrospective study was conducted in Oulu University Hospital in Northern Finland that has a

4 primary catchment area of 403000 inhabitants forming the hospital district. Being the largest

5 hospital in Northern Finland the university hospital also serves as tertiary referral center with a

6 geographical catchment area that comprises $49.5 \%$ of the total land area of Finland and $14 \%$ of

7 population. All neurocritical care, cardiac surgery, and the treatment of multi-traumas as well as

8 ECMO treatment of severe respiratory failure in Northern Finland, an area with 741000

9 inhabitants, is centralized in Oulu. This study focuses only on the primary catchment area

10 population.

\section{Ethics}

12 The study protocol was approved by hospital administration. The statement from the regional

13 ethics committee was not required due to the retrospective design of the study, the local policy 14 and Finnish law.

15 Demographic data including age, gender, chronic diseases, and medications, chronic alcohol abuse, and occupation were retrieved from the medical records and the patient data management system (PDMS). The causes of the admissions were retrieved from the electronic medical records.

18 The data concerning severity of illness, including Acute Physiology and Chronic Health Evaluation

$19 \quad\left(\right.$ APACHE II) ${ }^{7}$ and Sequential Organ Failure Assessment (SOFA) ${ }^{8}$ scores, the rooting of ICU

20 admission, ICU length of stay (LOS), and hospital mortality, were obtained from the PDMS.

21 Therapeutic Intervention Scoring System (TISS) ${ }^{9}$ was used to compare ICU resources between the 
1 groups. Alcohol abuse, unemployment, and retirement were recorded if these conditions were

2 mentioned in the medical records.

Residential area

Statistics Finland provides open data including median annual income per each inhabitant of each

7 postal code area in Finland. The postal code of each patient in the study population was retrieved

8 from the PDMS, and these postal codes were matched to the database of Statistics Finland. There

9 were admissions from 133 postal code areas with a total population of 284280 inhabitants aged 18 years or older, and 59800 of them were older than 65 years. The lowest median annual income of a postal code area of the patients in the study population was $€ 18979$ and the highest $€ 53864$. The postal codes were ranked according to the annual median income of the area by using the year 2013 as the index year. The study population was divided into three income area categorieslow-income area, middle-income area, and high-income area-according to the rank. Annual median income ranged in the low-income areas from $€ 18979$ to $€ 28841$, in the middle-income areas from $€ 28879$ to $€ 33856$, and in the high-income areas from $€ 34221$ to $€ 53864$. The median annual income in Finland in year 2013 was $€ 38775$ and $20 \%$ of the population had median annual income less than $€ 19200^{10}$. The residential area was considered urban if it was located to the centers or suburban areas of the main towns in the hospital district area. 
1 The data was analyzed using SPSS (IBM SPSS Statistics for Windows, Version 22.0, Armonk, NY).

2 Proportional data were expressed as numbers $(n)$ and percentages (\%). The incidences of annual

3 ICU admissions and crude ICU admission-related mortality were presented per 1000 inhabitants

4 per year with 95\% confidence intervals. The incidences between income areas were compared

5 using Poisson regression. Continuous data are expressed as medians and $25^{\text {th }}$ to $75^{\text {th }}$ percentiles

$6 \quad\left[25^{\text {th }}-75^{\text {th }} \mathrm{PCT}\right]$. Kaplan-Meier survival curves were drawn and comparison between curves was

7 performed using log-rank test. The categorical data was tested using Pearson's chi square and the

8 continuous variables were tested using non-parametric Kruskal-Wallis test. Two-tailed $P$-value less

9 than 0.05 was considered statistically significant.

Results

\section{Patients}

There were a total of 2158 ICU admissions during the year 2014. After excluding the admissions resulting from cardiac surgery $(n=631,29.2 \%)$, elective surgery $(n=329,15.2 \%)$, and trauma ( $n=213,9.9 \%)$, there were 985 admissions (45.6\%) that fulfilled the inclusion criteria for the study.

17 Of these, a total 735 admissions (34.1\% of all ICU admissions in 2014) were in patients older than

1818 years living in the hospital district area, and these were included in the study. The number of 19 individual patients was 687.

20 There were 245 admissions in each income category with 230 individual patients from low-income

21 areas and 226 and 231 patients from middle- and high-income areas, respectively. The number of residents in all the income areas combined was 284 280. The number of residents older than 65 
1 years was 59 800. The proportion of residents older than 65 years living in low- and middleincome areas was 41221 of 170894 (24.1\%) in contrast to 18579 of $113384(16.4 \%, P<0.001)$ in

3 high-income areas. The income groups did not differ in terms of gender, age, or severity of illness.

4 The middle-income areas were less urbanized compared with the low- and high-income areas

5 (Table 1). <insert table 1 here>

6 Psychiatric diagnosis was recorded in 37 of the 245 (15.1\%) patients from the high-income areas

7 when the corresponding rate in low- and middle-income areas combined was 96 of 490 (20.0\%,

$8 P=0.14)$. Daily psychotropic medication was recorded in 35 of $245(14.3 \%)$ of the patients from the

9 high-income areas in contrast to 101 of $490(20.6 \%, P=0.037)$ in the low- and middle-income areas

10 combined. The unemployment and retirement rate in the working-aged population was $22.9 \%$ (96

11 of 418 ) and the rate of chronic alcohol abuse was recorded in $34.6 \%$ of the admissions (254 of

12 735).

The causes of admissions

A total of 102 (41.6\%) of the 245 admissions from high income areas were admitted from emergency department (ED) when the corresponding rates in low- and middle-income areas were $128(52.2 \%)$ and $129(52.7 \%, P=0.022)$ (Table 1.)

The most common causes for the admissions were respiratory, neurological and cardiovascular causes. The causes of admissions did not differ between the income areas in patients aged between 18 and 65 years or in the patients older than 65 years. Respiratory causes were the most common causes in the group of younger patients and neurological causes among the older patients (Table 2). Poisonings were more common in low-income areas compared with middleand high-income areas in patients aged between 18 and 65 years ( 20 of 138 vs 25 of $280, P=0.04$ ). <insert table 2 here> 
The incidence of ICU admission

2 The incidence of ICU admission was 2.6 per 1000 inhabitants per year (95\% Cl, 2.4-2.8). The

3 incidence was lowest in working-aged population living in the high-income areas $(1.5[95 \% \mathrm{Cl}, 1.3$ -

$4 \quad 1.8])$ and highest in people older than 65 years living in the high-income areas (5.5 [95\% $\mathrm{Cl}$, 4.6-

5 6.7]). The incidence of ICU admissions in working-aged population was significantly lower in high-

6 income areas compared with middle-income areas $(p=0.001$ and $p=0.009)$ (Table 3). <insert table 3

7 here>

$8 \quad$ Outcome

9 During the 735 ICU admissions, death occurred in 59 patients (8.0\%), and 50 of the 676 ICU

10 survivors (7.4\%) died during the hospital stay. There were no differences in in-hospital mortality or

11 LOS between the income groups (Table 4). A total of 235 of the 687 patients (34.2\%) had died by

12 the end of the year 2015. There were no differences in mortality during the follow-up between the

13 income groups in total $(P=0.90)$, in working-aged population $(P=0.45)$ or in patients older than 65

14 years $(P=0.35)$ (Figure 1, Figure 2, Figure 3). <insert table 4. and figures here> 
1 The main finding of the present study is that there were significant differences in the incidence of

2 ICU admissions depending on the income of the residential area. This is a novel finding. The lowest

3 incidence was found among the 18- to 65-year-olds and the highest in persons older than 65 years,

4 both in the high-income residential area. The incidence in working-aged population was $25 \%$

5 higher in low- and middle-income areas compared with high-income areas. There were no

6 differences in short-term or long-term mortality between the areas.

7 Results from previous studies have indicated differences in case mix and outcome in ICU-admitted

8 patients from different social classes or ethnic backgrounds. ${ }^{1,2,6}$ In the present series from an area

9 with fully covering social insurance system, we showed a difference in the incidence of ICU

10 admissions, which was an unexpected finding. Persons aged between 18 and 65 years from low-

11 and middle-income areas were more often admitted to ICU compared with persons from high-

12 income areas. We did not find major differences in the patient demographics, except the lower

13 urbanization rate in the middle-income area, higher rate of use of psychoactive drugs and higher

14 rate of poisonings in the low-income areas and higher rate of admissions from ED in low-and

15 middle income areas, to explain the difference in the ICU admissions. Also, the severity-of-illness

16 scores were comparable between the patient populations from the three different income areas.

17 Although the number of inhabitants older than 65 years in the high-income area was smaller than

18 in the other two income areas, the incidence of intensive care admissions was the highest.

19 However, this was not significantly different from the incidence in the same population in the low-

20 and middle-income areas.

22 One explanation for the difference in the need of intensive care could be found in the unemployment and retirement rates. The difference in unemployment was not statistically 
1 significant, but there was a trend towards a higher rate on unemployment in the low- and middle-

2 income areas. Being included in the occupational health care may have improved disease

3 prevention in high-income areas and partly decreased the risk of critical illness. The higher rate of

4 ICU admissions from the ED in low-income areas suggests a limited access to occupational - and

5 primary health care services. It is notable that the unemployment was remarkably high in this

6 material, affecting $23 \%$ of the working-aged population, and therefore the role of occupational

7 health care in disease prevention was probably limited in this patient material. Secondly, there

8 was a difference between the income areas in the use of psychoactive medication and the

9 presence of psychiatric comorbidities in that both were more common in low- and middle-income

10 areas compared to high-income areas. Moreover, we found a lower rate of poisonings in the

11 middle- and high-income areas, indicating better psychiatric well-being of the population. It is

12 known that psychiatric conditions are linked to an increase in somatic comorbidities, which in turn

13 may lead to an increased need for intensive care. ${ }^{11,12}$ We did not include the cigarette smoking to the

14 collected data, which can be considered as a limitation since smoking habits may have an impact on the

15 general health of the population.

Thirdly, the middle-income areas were less urbanized compared with low- and high-income areas.

Some regional differences in morbidity and mortality have been reported. We have previously shown differences in trauma types and mortality in urban and rural areas in Northern Finland. ${ }^{13}$

Moreover, recent study from Northern Finland showed a decrease in the use of primary health care in population from lower social class and in populations living in the rural areas. ${ }^{14}$ The health care providers are usually located in urban centers and therefore the reachability of the health care services may be compromised, especially in the most rural areas. 
1 To our knowledge, this is the first study in Nordic countries that has aimed to evaluate differences

2 in the incidences of ICU admission between areas of different income. A large number of

3 admissions were included, and the study was performed in a country with fully social security

4 cover. Moreover, the population included in the study was located in an area where only one

5 hospital provides intensive care, which minimized the risk of selection bias. The results of the

6 present study are generalizable to areas where the health care system is paid for by the state. In

7 the light of the present results more effort should be made to prevent conditions leading to ICU

8 admissions in the low- and middle-income areas. The most significant differences between areas

9 were found in psychiatric comorbidity and poisoning-related admissions. Moreover, the high unemployment rate in the study population raises the question of the extent to which the lack of occupational health care may have affected the present results.

The present results suggest, that to decrease the need of intensive care admissions, a focus should be in reachability of primary health care services providing disease prevention and good care of chronic somatic and psychiatric conditions. Good somatic care including preventive measures aimed to chronic diseases, cigarette smoking, obesity and alcohol consumption as well as suicide prevention should be the focus.

\section{Limitations}

The main weakness of the present study is that we were not able to compare the incidences of ICU admission with incidences of hospital admission in the postal code areas included in the study.

Thus we cannot evaluate the use of general healthcare resources in this population that could have affected the ICU use. Secondly, we were not able to present individual annual income data that would have enabled us to study more homogenous patient groups; instead, we used the 
1 annual median income of the residential areas. However, the method of the present study has

2 been used in the previous studies. ${ }^{1,2}$ The resource planning in the health care system is based on

3 the residential areas instead of individual income and therefore this setting produces results that

4 have stronger clinical relevance. Furthermore, the categorization of patients into the income

5 categories was arbitrary. However, the median income of low- and middle income areas was

6 below the annual median income of Finnish population, which was $€ 38775$ in $2013 .{ }^{10}$

8 In summary, the incidence of ICU admission in working aged population was $25 \%$ higher in those

9 areas where the annual median income was below the annual median income in Finland. There were no differences in hospital or long-term mortality. Psychosocial comorbidities, including unemployment, use of psychoactive drugs, and chronic alcohol abuse, are common in patients admitted to ICU in Northern Finland. 


\section{References}

1. Hutchings A, Raine R, Brady A, Wildman M, Rowan K. Socioeconomic status and outcome from intensive care in England and Wales. Med Care 2004; 42:943-51.

2. Erickson SE, Vasilevskis EE, Kuzniewicz MW, Cason BA, Lane RK, Dean ML, Rennie DJ, Dudley RA. The effect of race and ethnicity on outcomes among patients in the intensive care unit: a comprehensive study involving socioeconomic status and resuscitation preferences. Crit Care Med 2011; 39:429-35.

3. Mendu ML, Zager S, Gibbons FK, Christopher KB. Relationship between neighborhood poverty rate and bloodstream infections in the critically ill. Crit Care Med 2012; 40:1427-36.

4. Epstein AM, Stern RS, Weissman JS. Do the poor cost more? A multihospital study of patients' socioeconomic status and use of hospital resources. N Engl J Med 1990; 322:1122-8.

5. Brattstrom O, Eriksson $M$, Larsson $E$, Oldner A. Socio-economic status and co-morbidity as risk factors for trauma. Eur J Epidemiol 2015; 30:151-7.

6. Ho KM, Dobb GJ, Knuiman M, Finn J, Webb SA. The effect of socioeconomic status on outcomes for seriously ill patients: a linked data cohort study. Med J Aust 2008; 189:26-30.

7. Knaus WA, Draper EA, Wagner DP, Zimmerman JE. APACHE II: a severity of disease classification system. Crit Care Med 1985; 13:818-29.

8. Vincent JL, Moreno R, Takala J, Willatts S, De Mendonca A, Bruining H, Reinhart CK, Suter PM, Thijs LG. The SOFA (Sepsis-related Organ Failure Assessment) score to describe organ dysfunction/failure. On behalf of the Working Group on Sepsis-Related Problems of the European Society of Intensive Care Medicine. Intensive Care Med 1996; 22:707-10.

9. Cullen DJ, Civetta JM, Briggs BA, Ferrara LC. Therapeutic intervention scoring system: a method for quantitative comparison of patient care. Crit Care Med 1974; 2:57-60.

10. Statistics Finland: Annual income in Finland 2013. Tilastokeskuksen PX-tietokannat 2015. http://pxnet2.stat.fi/PXWeb/pxweb/fi/StatFin/StatFin_tul_tjkt/050_tjkt_tau_105.px/table/ tableViewLayout1/?rxid=828c6445-9639-445c-8df8-b7809d6fa15d

11. Cook JA, Razzano LA, Swarbrick MA, Jonikas JA, Yost C, Burke L, Steigman PJ, Santos A. Health risks and changes in self-efficacy following community health screening of adults with serious mental illnesses. PLoS ONE 2015; 10:e0123552.

12. Razzano LA, Cook JA, Yost C, Jonikas JA, Swarbrick MA, Carter TM, Santos A. Factors associated with co-occurring medical conditions among adults with serious mental disorders. Schizophr Res 2015; 161:458-64.

13. Raatiniemi L, Steinvik T, Liisanantti J, Ohtonen P, Martikainen M, Alahuhta S, Dehli T, Wisborg T, Bakke HK. Fatal injuries in rural and urban areas in northern Finland: a 5-year retrospective study. Acta Anaesthesiol Scand 2016; 60:668-76. 
9 <Figure 1. The Kaplan-Meier survival curves of the 687 non-trauma patients admitted to ICU>

14. Lankila T, Nayha S, Rautio A, Rusanen J, Taanila A, Koiranen M. Is geographical distance a barrier in the use of public primary health services among rural and urban young adults? Experience from Northern Finland. Public Health 2016; 131:82-91.

10 <Figure 2. The Kaplan-Meier survival curves of the 387 non-trauma patients aged 65 years or

11 younger admitted to ICU>

12 <Figure 3. The Kaplan-Meier survival curves of the 300 non-trauma patients older than 65 years 13 admitted to ICU> 